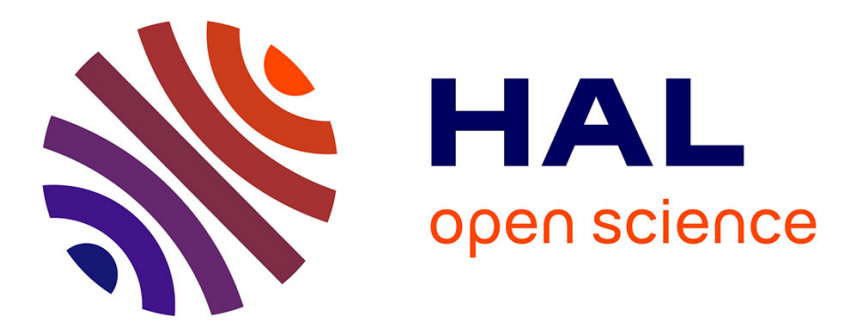

\title{
Organic nanocrystals grown in sol-gel matrices: new hybrid organic-inorganic materials for optics
}

\author{
Virginie Monnier, Emilie Dubuisson, Nathalie Sanz, Alain Ibanez
}

\section{To cite this version:}

Virginie Monnier, Emilie Dubuisson, Nathalie Sanz, Alain Ibanez. Organic nanocrystals grown in solgel matrices: new hybrid organic-inorganic materials for optics. Research on Chemical Intermediates, 2008, 34, pp.155 - 167. 10.1163/156856708783623492 . hal-01846291

\section{HAL Id: hal-01846291 \\ https://hal.science/hal-01846291}

Submitted on 23 Jul 2018

HAL is a multi-disciplinary open access archive for the deposit and dissemination of scientific research documents, whether they are published or not. The documents may come from teaching and research institutions in France or abroad, or from public or private research centers.
L'archive ouverte pluridisciplinaire HAL, est destinée au dépôt et à la diffusion de documents scientifiques de niveau recherche, publiés ou non, émanant des établissements d'enseignement et de recherche français ou étrangers, des laboratoires publics ou privés. 


\title{
Organic nanocrystals grown in sol-gel matrices: new hybrid organic-inorganic materials for optics
}

\author{
VIRGINIE MONNIER, EMILIE DUBUISSON, NATHALIE SANZ \\ and ALAIN IBANEZ*
}

Institut Néel, CNRS, UPR 2940, associé à l'Université J. Fourier, BP 166, 38042 Grenoble cédex 9 , France

Received 25 November 2006; accepted 20 January 2007

\begin{abstract}
We have developed a simple and generic preparation of stable organic nanocrystals grown in gel-glass matrices. The synthesis of these hybrid organic-inorganic materials is based on the confined nucleation and growth of organic phases in the pores of dense gels. For bulk nanocomposite samples, narrow size distributions of particles (10-20 nm in diameter) are obtained. We have extended this method to the preparation of organic nanocrystals embedded in sol-gel thin films prepared by spin-coating. For all these nanocomposite materials, we have significantly increased the dye stability and obtained promising optical properties: luminescence, non-linear optical properties or photochromism. Moreover, we have also demonstrated basic working principles of a new type of fluorescent nanosensor through the preparation of organic luminescent nanocrystals grown in silicate films.
\end{abstract}

Keywords: Nanocrystal; sol-gel; hybrid organic-inorganic materials; nanocomposite; optics.

\section{INTRODUCTION}

Organic bulk crystals exhibit unique optical properties, such as luminescence [1], photochromism [2], or two-photon absorption [3]. Moreover, the structural flexibility of organic molecules and the possibility of precise and adequate organic synthesis assisted by predictive molecular-engineering rules is a fascinating asset of organics in contrast with the more rigid nature of inorganics. Nevertheless, the thermal and mechanical stabilities of organic single crystals remain limited: thermal decomposition around or lower than $200^{\circ} \mathrm{C}$ and mechanical hardness below

\footnotetext{
*To whom correspondence should be adressed. Tel.: (33-4) 7688-7805; Fax: (33-4) 7688-1038; e-mail: alain.ibanez@grenoble.cnrs.fr
} 
that of $\mathrm{KH}_{2} \mathrm{PO}_{4}$ (150 Vickers) which represents the limit of convenient slicing and polishing of the crystals. This weak crystal stability is due to the involvement in all these crystal structures of van der Waals or hydrogen bonds for the cohesion of the three-dimensional packing. Thus, their industrial development is limited due to delicate shaping and processing of optical devices.

In order to overcome this basic mechanical drawback of organic bulk crystals, since 1997 we have undertaken another strategy on hybrid materials based on the confined nucleation and growth of dyes in the pores of sol-gel matrices [4-6]. We selected silicate gel matrices for the high flexibility of the corresponding sol-gel chemistry based on silicon alkoxide precursors and also for its ability to obtain stable inorganic matrices close to the room temperature. Thus, the solgel chemistry is compatible with a wide variety of organic molecules. Since 1984 [7] it has been shown that the sol-gel method can be used to encapsulate organic molecules in inorganic matrices, bulk or thin films. In all these previous works, dyes were dispersed within or grafted onto sol-gel networks [7-9]. In our works, the molecules are aggregated through their confined nucleation in the pores of gel frameworks. This allows us to prepare a new type of hybrid organicinorganic materials constituted by organic nanocrystals grown in silicate bulky gel-glasses or thin films. These nanocomposite compounds not only combine the optical properties of organic crystals (luminescence, non-linear optical properties, photochromism, etc.) with those of amorphous inorganic materials (high stability, convenient processing and shaping and good optical properties [10]), but also the advantages of nanocrystals: their small size leads to the preparation of non-diffusing materials for visible and IR wavelengths. Also, through dye nanocrystallisation, the chemical stability and photostability of the organic phase are also improved in comparison to isolated molecules dispersed in solutions or in solid matrices such as sol-gel or polymers.

For the moment, in contrast to metallic or semiconductor nanoparticles [11], organic nanocrystals have attracted little attention due to their delicate processing and shaping and to difficult structural characterizations of small and brittle particles constituted of light chemical elements. Previously, only a few preparations of organic nanocrystals have been carried out in solution [12-15]. The resulting isolated nanocrystals should then be dispersed in matrices through another delicate step to prepare suitable optical devices while in our preparation method final optical materials are directly obtained.

In this paper, we introduce the principles of our generic nanocrystallisation process of organics in dense sol-gel matrices. We briefly present the preparation, characterization and the optical properties of organic nanocrystals grown in bulk gel-glasses. Finally, we report more in details recent results obtained on the nanocrystallisation of organics in sol-gel thin films. 


\section{ORGANIC NANOCRYSTALS GROWN IN BULK GEL-GLASSES}

The preparation of organic nanocrystals in bulk gel-glasses is based on the control of nucleation and growth kinetics of the dye confined in the pores of dense gels. This nanocrystallization method can be decomposed in well-separated steps:

It starts from homogenous solutions containing a solvent, the organic phase, the silicon alkoxide precursors of the gel and a small amount of water for the hydrolysis of the alkoxides. These solutions are inserted in silica airtight cells and heated at $80^{\circ} \mathrm{C}$ in order to rapidly dissolve the dye.

Within a few minutes, transparent sols are obtained giving wet gels after several hours. These gels are then aged for 1-2 days according to the type of the sol-gel matrix. This syneresis step allows to adjust the gel porosity and avoids the formation of cracks during the drying of the samples.

The nucleation of the organic phase is induced in the pores of the gel that act as nanometer-scale reactors for crystal growth in solution. This nucleation in confined solutions is only possible at very high supersaturations, since the mass transport only occurs by diffusion [16, 17]. Such high supersaturations are achieved by dropping the temperature from $80^{\circ} \mathrm{C}$ to ambient or chilling temperatures. The relative supersaturation, $\sigma$, depends on the concentration of organic molecules, their solubility in the solvent and the chemical nature of the host matrix. Typically, we apply relative supersaturations ranging between 100 and $200 \%$. These very high $\sigma$ values, at the initial stage of the process, lead to the rapid formation of a high number of organic nuclei in the interconnected pore network of the gel. This burst of nucleation leads to a decrease of $\sigma$, stops the nucleation step rapidly and limits the subsequent growth stage.

Then, the gels are slowly dried during several days or weeks at room temperature to avoid typical problems of cracks on the resulting gel-glasses. During this drying step, the growth rate of the nuclei is strongly reduced by the slow diffusion coefficients of molecules in the dense gel matrices.

Finally, the samples are annealed in air or under inert gas (depending on the chemical stability of the organic phase) at a temperature just below the melting point of the dye to improve the crystallinity of the particles. These annealings allow also to remove the residual solvent and to stabilize the nanocomposite materials.

Our control of the confined nanocrystallization of the organic phases is based on the diagram established by La Mer for the preparation of monodisperse crystals in free solutions [18]. The dense gel matrices exhibit three main advantages referred to free solutions. The confined nucleation, only possible at very high supersaturations, favours the formation of a single "burst" of nuclei; the slow growth of the particles in the dense gel matrix is negligible in comparison to the nucleation rate and finally the gel prevents nanocrystals from coalescing. The adjustment of the experimental conditions of nanocrystallization is well illustrated in Fig. 1. When a high supersaturation of the organic phase is applied to a gel of low density, just after gelation, the growth rate is too high and leads to dendritic growth (Fig. 1a). When the nucleation is induced after a syneresis step of a few hours, the matrix is 

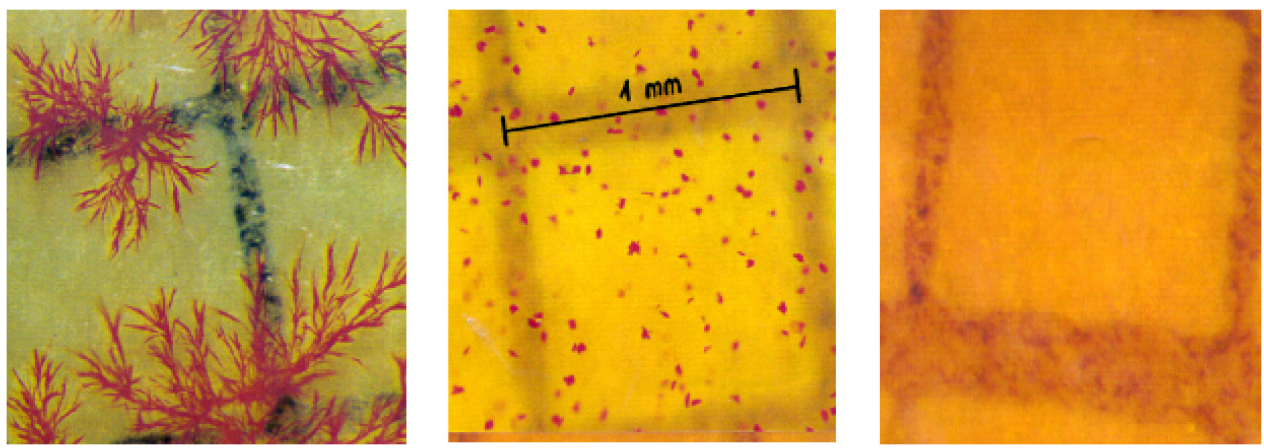

Figure 1. Confinement of nucleation and growth of the organic phase versus the density of the wet gel matrix. This figure is published in colour at http://www.ingenta.com

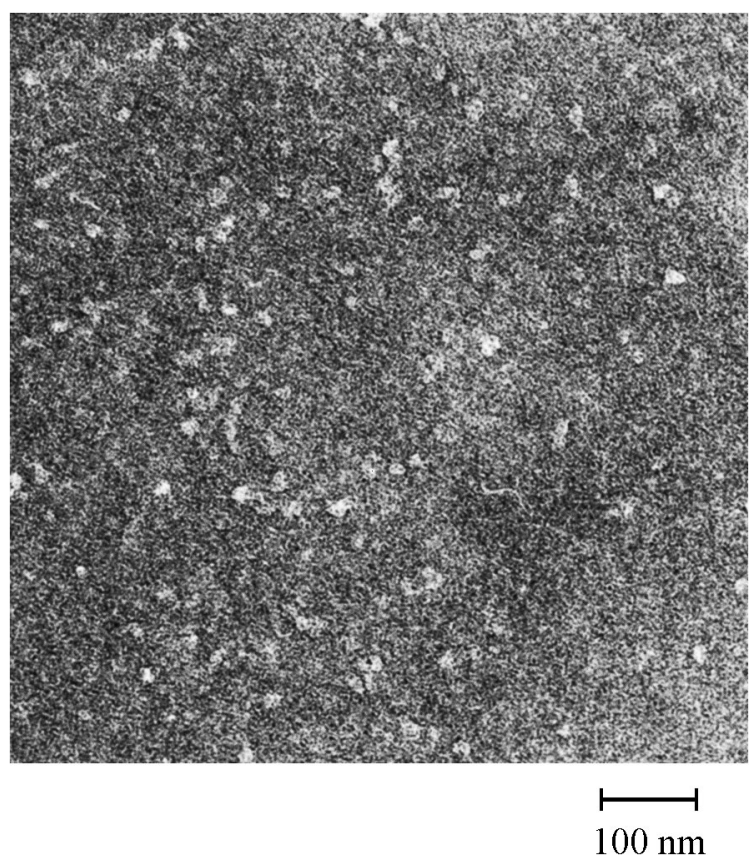

Figure 2. Transmission electron microscopy image exhibiting organic nanocrystals well-dispersed in the gel-glass matrix with a narrow size distribution $(10-20 \mathrm{~nm})$.

not very dense and the particle growth is not negligible referred to the nucleation rate. This leads to the formation of polydisperse microcrystals ranging from 20 to $50 \mu \mathrm{m}$ in diameter (Fig. 1b). Finally, when the high supersaturation is applied on dense matrices, 1-2 days after gelation, the fast nucleation is well dissociated to the slow diffusion-controlled particle growth. Thus, original nuclei can grow uniformly when nucleation is over to yield monodisperse nanoparticles (10-20 nm, see TEM photograph in Fig. 2) and transparent samples (Fig. 1c). 
In order to determine the role of the host framework on the aggregation of the organic molecules, different silicon alkoxide precursors have been used for the preparation of the gel glass. We used tetramethoxysilane (TMOS) and tetraethoxysilane (TEOS). After these initial experiments, TMOS was selected for its gelation time which is shorter than that of TEOS. Silanol substituents of the gelled TMOS (or TEOS) can form hydrogen bonds with the organic molecules. These interactions between molecules and the silicate network disturb the dye aggregation. To minimize these interactions, methyltrimethoxysilane (MTMOS) was added to the TMOS precursor. Gel glasses of high optical quality are obtained from TMOS + MTMOS equimolar mixtures. The non-bridging methyl function, arising from MTMOS, covers the gel pores [19] and only forms van der Waals bonds with surrounding molecules. Thus, the addition of MTMOS reduces the strength of the chemical interactions of the gel with organic molecules and facilitates the growth of dye clusters having a critical radius for the nucleation of nanocrystals.

Typical gels were prepared according to literature procedures [10]. Gelation occurred after a few hours from a one-step hydrolysis and condensation. These reactions were carried out under acidic $(\mathrm{HCl})$ conditions with $1 \mathrm{H}_{2} \mathrm{O}$ molecule per $-\mathrm{OCH}_{3}$ function $\left(h=\left[\mathrm{H}_{2} \mathrm{O}\right] /\left[-\mathrm{OCH}_{3}\right]=1\right)$. Neutral conditions were also used for Lewis base organic molecules. In this case, in order to obtain transparent matrices, the TMOS precursor was mixed in equimolar proportions with methyldimethoxysilane (MDMS), which, like MTMOS, has one non-bridging $\mathrm{CH}_{3}$ function. For these 1:1 mixtures of TMOS + MDMS, 0.2 mol water per mol $-\mathrm{OCH}_{3}$ function is initially added in the sols and some water is added after $24 \mathrm{~h}$ to bring the $\mathrm{H}_{2} \mathrm{O} /-\mathrm{OCH}_{3}$ molar ratio up to 1 .

Depending on the nature of the dye, methanol, ethanol, dioxane, dimethylformamide, toluene, dimethylsulfoxide (DMSO) or tetrahydrofuran (THF) were used as solvents. Miscibility of these solvents with silicon alkoxides and water provided homogeneous initial solutions which were then used to dissolve the organic powder. The solvent concentration was specified by the molar ratio $s=$ solvent/alkoxide. In this study, all the monolithic samples were prepared with $s=1$. As for all types of crystal growths in solution it is necessary, for each chromophore, to specify the best solvent and the corresponding solubility evolutions with temperature, between room temperature and $80^{\circ} \mathrm{C}$.

Several organic molecules were chosen in order to demonstrate the flexibility of our generic process. We first selected 4-(N,N-diethylamino)-b-nitrostyrene (DEANST) and 1,4-di(2,5-phenyloxazole)benzene (POPOP) [4] for their non-linear optical and luminescence properties [20,21]. Because of the presence of $\mathrm{Cl}$ and $\mathrm{S}$ atoms, 2-amino-5-nitropyridium chloride (2A5NPCl) [22] and 4,4-sulfonyldiphenol (SDPH) were also used to improve the contrast in transmission electron microscopy (Fig. 2) [4]. In this work, we have characterized organic aggregates of $\alpha$-[(4'-methoxyphenyl) methylene]-4-nitro-benzene-acetonitrile (CMONS) [23] and stilbene 3 [24], which were selected for their luminescent and optical limiting properties, respectively. The concentration of the organic particles depends on their solu- 
bility and on the nature of the matrix. High doping levels, $l$, have been achieved with highly soluble molecules such as $\operatorname{SDPH}(l \approx 20 \mathrm{wt} \%)$ and stilbene $3(l \approx 30 \mathrm{wt} \%)$.

Using differential scanning calorimetry, we have recorded the melting point of nanocrystals of several different molecules. The strong endothermal peak corresponding to the melting of the dye proves the crystallinity of organic nanoparticles existence in the amorphous matrix. Moreover, generally the nanocrystals melt at a temperature significantly above the melting point of the microcrystalline powder of the pure dye. For example, NPP nanocrystals embedded in transparent xerogels melt a temperature $51^{\circ} \mathrm{C}$ higher than NPP powder (Fig. 3). This enhancement of thermal stability is due to the strong confinement of organic nanocrystals in the silicate matrix and to dye-matrix interactions (hydrogen bonds).

Thermogravimetric analysis reveals that no organic material is lost as the xerogels are heated above the melting point of the dye. The formation of fluorescent organic particles could also be followed by luminescence spectroscopy when the emission spectrum of dissolved molecules such as DEANST is significantly different from that of crystalline aggregates [4]. Particle sizes, which are dependent on experimental conditions (supersaturation, gel porosity, etc.) are fairly monodisperse. Nanocrystals of SDPH or 2A5NPCl have diameters between 20 and $100 \mathrm{~nm}$ [4]. Degree of crystallinity of aggregates cannot be determined using electron diffraction because organic materials are damaged under the focused electron beam when they are nanocrystals in xerogels or microcrystalline powders. In order to improve the stability of organic nanocrystals, future TEM studies will be conducted using samples maintained at the temperature of liquid nitrogen. X-ray diffraction also failed to reveal the degree of crystallinity of aggregates because of low scattering factors and concentrations of the organic phases relative to those of the inorganic matrix.

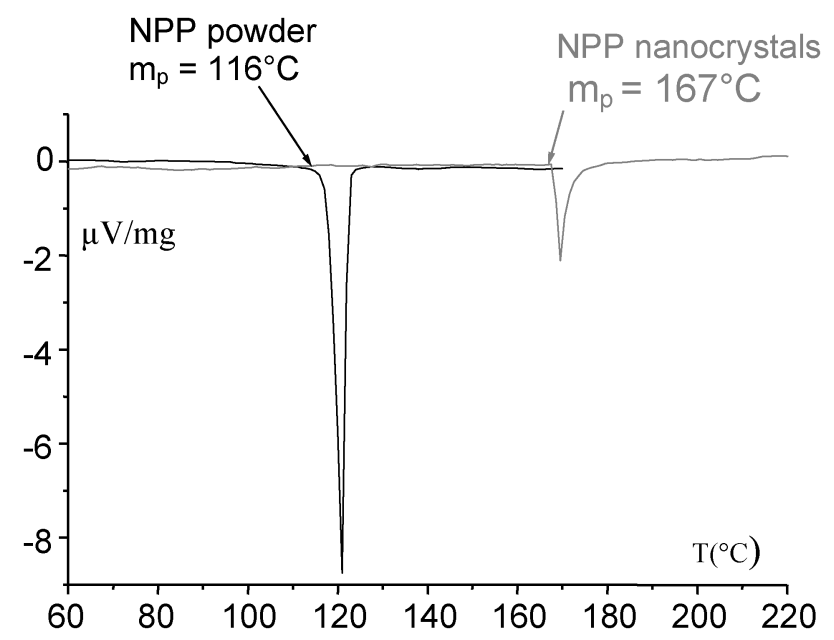

Figure 3. DSC curves of NPP powder and NPP nanocrystals grown in a gel glass. 


\section{ORGANIC NANOCRYSTALS GROWN IN SOL-GEL THIN FILMS}

We have extended our nanocrystallization process of organic phases in sol-gel matrices to the preparation of nanocomposite thin films [5]. These coatings are deposited by spin-coating on several types of substrates such as microscope slides, silicon wafers or polymers. As for the preparation of monolithic samples mixtures of silicon alkoxydes, solvent, water and organic phase are first inserted in airtight containers and heated for about $10 \mathrm{~h}$ at $80^{\circ} \mathrm{C}$ in order to obtain homogeneous sols. Sols are then aged during several days at room temperature. This aging step is necessary to obtain high quality films and to ensure the control of the particle growth.

In the preparation of organic nanocrystals grown in bulk silicate matrices, matrix gelation, nucleation and particle growth are well-separated steps. In contrast, for the thin-film process, nucleation and growth of the organic phase begin in the viscous sol at the first stage of the coating process, when droplets of solution are spread out on the substrate. The fast evaporation of the solvent induces instantaneously a high supersaturation of the dye which is associated with the polycondensation of the silicate framework. Thus, we have a one-step process, since nucleation and growth of the organic particles occur simultaneously with the formation of the solgel matrix which avoids the coalescence of the organic nanocrystals. The coatings are then stabilized by annealing under inert gas at a temperature just above the melting point of the dye.

This preparation of nanocomposite coatings involves strong coupling effects between the elementary processes of this method: nucleation and growth of the organic particles and polymerization of the silicate matrix. Preparing monodisperse particles requires a high control of these coupling effects. Wide distributions of particle size arise generally from nucleation over a relatively long period of time, when young nuclei are produced during the growth of older nuclei. Here we take advantage of the very high supersaturation achieved through the rapid evaporation of the organic solvent. This evaporation induces the germination of a high number of nuclei. The instantaneous nucleation quickly lowers the concentration of the molecularly dispersed dye below the critical concentration for self-nucleation, so only a single "burst" of nuclei occurs, leading to narrow size distributions of nanocrystals (Figs 4b and 5).

Processing of nanocomposite coatings is mainly controlled by the chemistry of the sol-gel precursors, the dye concentration, the nature and volatility of the solvent and finally by the rotation speed of the spin-coating apparatus. As for the preparation of bulk samples, we have first used TMOS and TEOS silicon alkoxides. We have then particularly selected the equimolar mixture of TMOS + MTMOS. Indeed, as for bulk samples, non-bridging methyl substituents of MTMOS cover the gel pores and facilitate the dye aggregation as illustrated in the next section. The hydrolysis and polycondensation reactions are acid catalyzed $(\mathrm{HCl})$ with $h=\left[\mathrm{H}_{2} \mathrm{O}\right] /\left[-\mathrm{OCH}_{3}\right]=1$. Depending on the organic phase, methanol, ethanol, 1,4-dioxane, ethyl acetate or THF are used as solvent with the molar ratio $s=3-6$. 


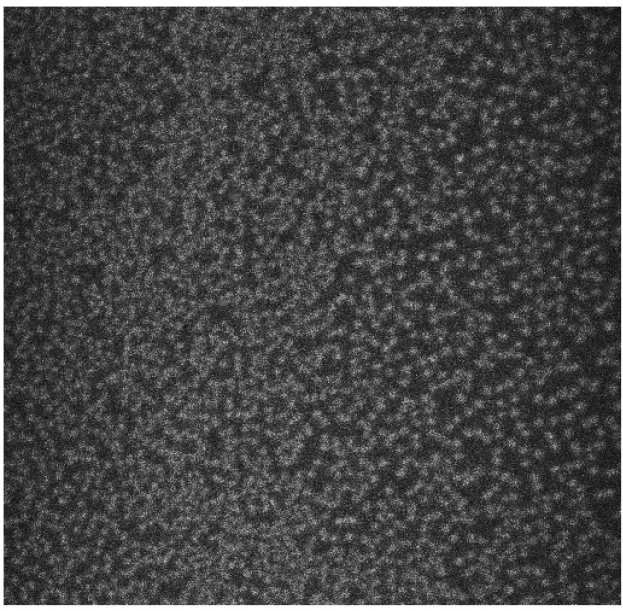

$\overline{2 \mu \mathrm{m}}$

(a)

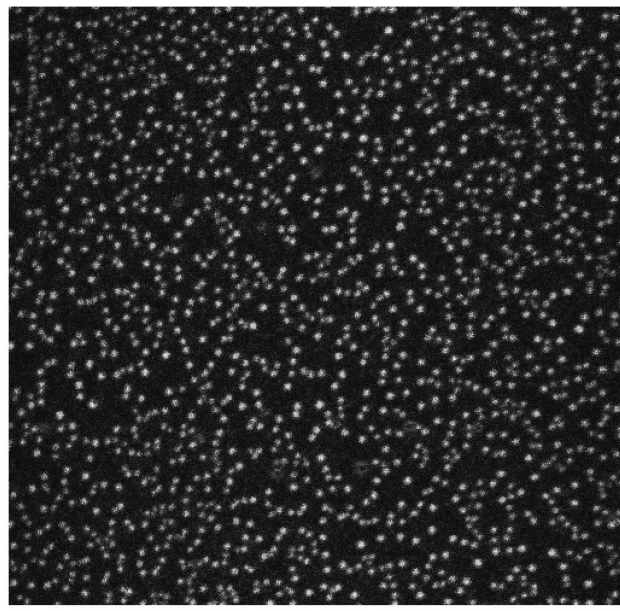

(b)

Figure 4. Confocal microscope images of DEANST nanocrystals grown from MTMOS gel precursor (a) and from an equimolar mixture of TMOS and MTMOS alkoxides (b).

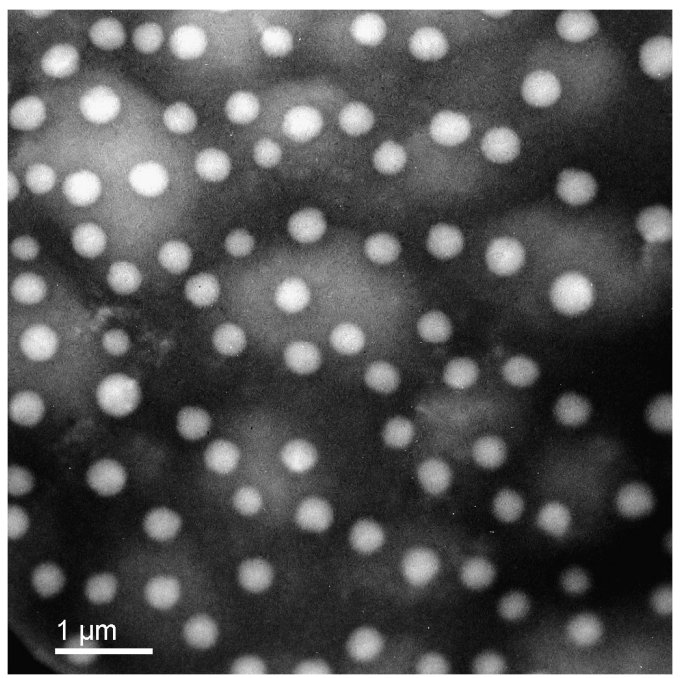

Figure 5. TEM image of rubrene nanocrystals $\left(d=1 \times 10^{-3}\right)$ in a TMOS + MTMOS (1:1) sol-gel thin film and corresponding size distribution.

This molar ratio, directly related to the viscosity of the initial sol, is adjusted to achieve the good nanocrystallization conditions and the preparation of high quality coatings [5]. As for the preparation of bulk nanocomposite samples, in order to demonstrate the validity and the flexibility of this nanocrystallisation process, we have grown nanocrystals of several organic phases such as DEANST and CMONS, chosen for their luminescent and non-linear optical properties [20, 23], NPP for its high quadratic NLO efficiency [25], and pyrido-spiropyran (PSP) for 
photochromism properties [26]. We have also grown other types of nanocrystals, such as naphthalene, to demonstrate the gain in stability of the organic phase through the nanocrystallisation process, as pure naphthalene crystals exhibit a high sublimation rate. The concentration of the organic phase depends on its solubility in the corresponding solvent and on the chemistry of the host matrix. The doping level is expressed as the molar ratio $d=$ organic/alkoxide $\approx 10^{-2}-10^{-1}$. Viscous sols are spread onto the substrates by spin-coating with rotation speeds between 2000 and $4000 \mathrm{rpm}$. In this way, films of $0.5-1 \mu \mathrm{m}$ thickness are prepared. We have adjusted the experimental conditions to obtain particles of sizes ranging between 100 and $500 \mathrm{~nm}$ in order to facilitate their observation by optical confocal microscopy.

\section{CHARACTERIZATION OF THE NANOCOMPOSITE COATINGS}

Optical confocal microscopy allowed us to have a direct observation of organic particles imaging their fluorescence or reflection. We used a confocal scanning microscope because it allows to eliminate the out-of focus blur and exhibits an improved lateral $(0.2 \mu \mathrm{m})$ and axial resolution $(0.8 \mu \mathrm{m})$. Images presented here were obtained by recording the fluorescence of DEANST nanocrystals (centered at $\lambda=640 \mathrm{~nm})$ excited with a He-Ne laser $(\lambda=633 \mathrm{~nm})$. As we will see, they clearly evidence the influence of the different elaboration parameters (matrix type, dye concentration, $d$, etc.) on the nanocomposite coatings.

For example, Fig. 4 shows confocal microscope images of DEANST nanocrystals grown in spin coated films using ethanol as solvent. When TMOS is the only gel precursor used (Fig. 4a) the DEANST luminescent particles in the coating are not as well-defined as when MTMOS is added (Fig. 4b). This is due to the non-bridging methyl functions of the host matrix, originating from MTMOS alkoxide, which lower the strength of dye-matrix interactions and, hence, favour the dye aggregation. Thus, well-dispersed particles with narrow size distributions, between 200 and $300 \mathrm{~nm}$ in Fig. 4b, are obtained with equimolar TMOS + MTMOS coatings.

On the other hand, when the rotation speed of the spin-coating apparatus is increased, the rate of solvent evaporation is increased (time confinement). Thus, the supersaturation applied on the organic phase and the formation rate of the gel framework increase. This decreases the process duration and leads to smaller particle sizes and narrower size distributions.

Another important parameter is the nature of the solvent [5]. Its volatility is directly related to the applied supersaturation while strong dye-solvent interactions can disturb the crystallization of the organic phase as in the case of strong dyematrix interactions. On the other hand, the size of the particles decreases directly with the dye concentration [5]. Thus, transparent thin films in the visible region can be prepared with nanocrystal sizes less than $200 \mathrm{~nm}$. In this case particles are observed by scanning electron microscopy (Fig. 5). 
Finally, atomic force microscopy shows that thin fims exhibit a good surface quality with very low roughness of around 10-20 A, because nanocrystals are well embedded in the sol-gel coatings [5]. Indeed, organic nanocrystals form monolayers in the middle of the film thickness [4] and, thus, are well stabilized by the surrounding inorganic matrix. The resulting improved stability of the organic phase is illustrated by the fact that nanocrystals of naphtalene do not sublime significantly from the matrix. Indeed, similar confocal microscopy images are obtained several months after the coatings' preparation.

Targeted optical properties of these nanocomposite films are nanocrystal spectroscopies (luminescence [27], photochromism [28] and non-linear optics [29]) and quenching of luminescence related to specific chemical or biological surroundings for fabrication of sensors. On the other hand, we have controlled of the spatial distribution of the nucleation sites of organic nanocrystals. Thus, we have obtained welldefined 2D and 3D arrays, through a multilayer process, of luminescent nanocrystals (Fig. 6) $[30,31]$ in order to obtain potential rewritable and volumic optical storage devices.

Recently, we have prepared various nanocrystal types to test their ability as chemical and biological sensors [32]: cyanomethoxynitrostilbene, naphtalimide, polyaromatic molecule (rubrene, tetracene) and perylene diimides. All these molecules are strongly fluorescent in the crystal state. In organic nanocrystals,

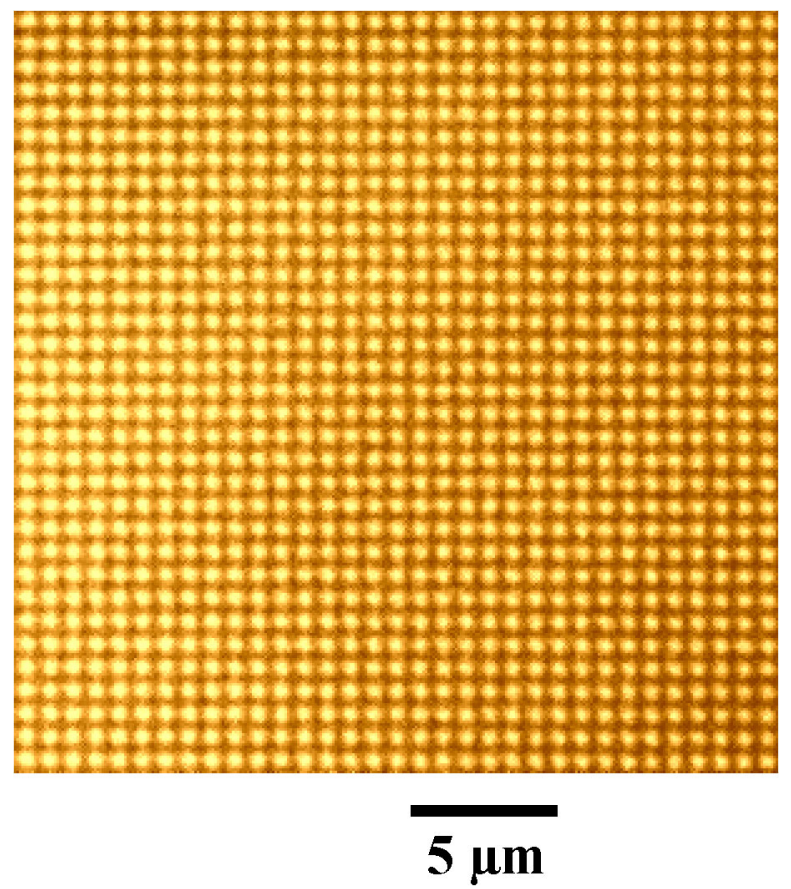

Figure 6. Two-dimensional array of luminescent nanocrystals grown on nanostructured substrate. This figure is published in colour at http://www.ingenta.com 
the fluorescence emission is spread around an assembly of chromophores $\left(10^{5}-\right.$ $10^{6}$ molecules), which are quenched simultaneously. Indeed, in these nanocrystals, molecules are weakly coupled. This limits the excitation trapping in the defects while crystal order allows to extend the excitation delocalisation. This should lead to an ultrasensitive detection. Moreover, these nanocrystals exhibit a significantly higher brightness and a better photostability than organic molecules dispersed in solution or in polymer matrix [2]. On the other hand, the open porosity of the solgel coatings is flexible from around 1 nanometer (microporosity) to several tenths of nanometers. This versatibility allows to adjust nanocrystals reactivity with their chemical environment. Thus, these porous matrices can act as a filter in size, charge and polarity to prevent from non-specific interactions with the environment.

In a first step, we have used as a molecular probe a coloured indicator (Cibacron blue and methylene blue (MB)) dissolved at a very low concentration in aqueous solutions spread onto sol-gel films. Our first results show that these coloured indicators diffuse inside the sol-gel matrix and adsorb at the organic nanocrystals surface. The fluorescence of organic nanocrystals is quenched through the adsorption of probe molecules on their surface. This strong effect is due to the large delocalization of the initial excitation in the whole nanocrystal which can reach the quenching site, that is, a probe molecule adsorbed at the nanocrystal surface. This energy transfer between nanocrystal molecules is similar to a single chromophore excitation. Therefore, a $\mathrm{NC}$ acts like a single light emitter. In the example shown in Fig. 7, MB is a redox indicator which is blue in presence of oxygen and colourless in the presence of a reducing sugar. When rubrene nanocrystals are in presence

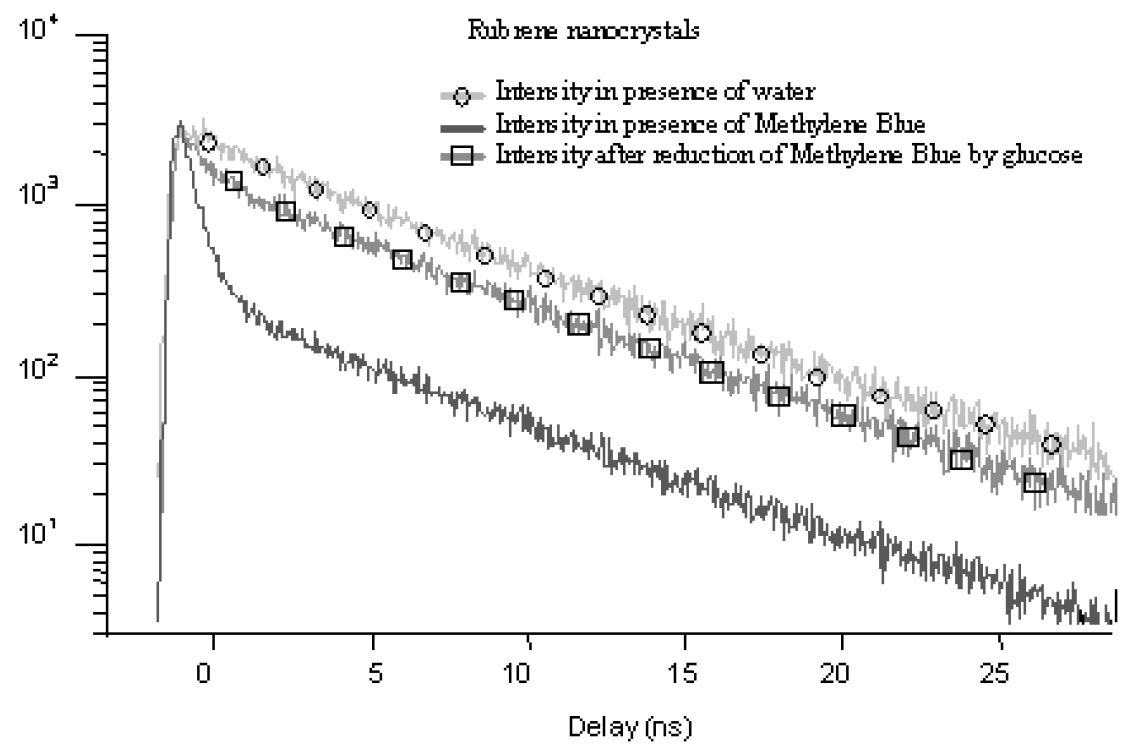

Figure 7. Fluorescence decays of rubrene nanocrystals in presence of water, MB and MB reduced by glucose. 
of MB (probe molecule) under its oxidized form (blue), an energy transfer occurs between the probe and the nanocrystals. This leads to a significant change of the fluorescence decay curve, while, if the MB is reduced (colourless form) by glucose (target molecule), the energy transfer between the probe and the nanocrystals does not occur and one finds again the fluorescence decay of rubrene nanocrystals.

Moreover, we demonstrate that this new type of nanosensor works by using various colored indicators (probes) which allow to excite the nanocrystals fluorescence depending on the chemical surrounding [29]. Thus, we can conceive a generic process to prepare various sensors for a same nanocrystal by using different molecular probes. Finally organic nanocrystals exhibit generally monoexponential fluorescence decays while those of inorganic particles (semiconductor nanocrystals) are multiexponential. Finally, work is in progress to open significantly the pore size of the NC matrices for the development of biological sensors.

\section{CONCLUSION}

We have designed a new type of hybrid organic-inorganic materials through a flexible process based on the confinement in time of the nucleation while the nanocrystal growth is controlled by the spatial confinement in the pores of the sol-gel network. These materials combine the optical properties of molecular crystals with the stability of the inorganic matrices, but also the advantages of nanocrystals: preparation of low diffusing samples and higher photostability than dispersed molecules. The validity of this process has been confirmed by using various dyes, solvents and sol-gel precursors. Narrow nanocrystal size distributions are obtained in bulk and thin films nanocomposites through the high control of polycondensation of the silicate matrices and the confined nucleation and nanocrystal growth of the organic phase. We have greatly improved the stability (mechanical, thermal, chemical and photochemical) of the organic crystals through their strong confinement in the pores of dense sol-gel matrices. Our optical characterizations show interesting properties such as quadratic NLO, electro-optic, optical data storage devices or optical power limiters. We have also demonstrated the basic working principles of fluorescent organic nanocrystals as nanosensors by adsorbing indicators on them. The signalization function is based on polyaromatic nanocrystals which exhibit a good stability and photostability when compared to typical dispersed dyes and a simple fluorescence signature (monoexponential decay) in comparison with inorganic nanocrystals. Finally, this new type of nanosensors exhibits other significant advantages such as low preparation cost, easy nanocrystal functionalizations with the possibility to use a lot of indicators developed in analytical chemistry [9]. Moreover, the sol-gel matrices can play the role of a filter in size (pores), in charge and polarity. So far, we have restricted our choice to organic molecules with optical properties, but similar hybrid nanocomposite materials could be designed with molecules exhibiting other specific properties such as magnetism. 


\section{REFERENCES}

1. N. Tessler, G. J. Denton and R. H. Friend, Nature 382, 695 (1996).

2. M. Irie, S. Kobatake and M. Horichi, Science 291, 1769 (2001).

3. J. M. Nunzi and F. Charra, Pure Appl. Opt. 7, 501 (1998).

4. A. Ibanez, S. Maximov, A. Guiu, C. Chaillout and P. L. Baldeck, Adv. Mater. 10, 1540 (1998).

5. N. Sanz, A. C. Gaillot, P. L. Baldeck and A. Ibanez, J. Mater. Chem. 10, 2723 (2000).

6. N. Sanz, P. L. Baldeck and A. Ibanez, Synth. Met. 115, 229 (2000).

7. D. Avnir, D. Levy and R. Reisfeld, J. Phys. Chem. 88, 5957 (1984).

8. D. Avnir, V. R. Kaufman and R. Reisfeld, J. Non. Cryst. Solids 74, 395 (1985).

9. C. Sanchez and F. Ribot, New J. Chem. 18, 1007 (1994).

10. C. J. Brinker and G. W. Scherer, Sol-Gel Science, The Physics and Chemistry of Sol-Gel Processing. Academic Press, New York, NY (1990).

11. R. Reisfeld, in: Structure and Bonding, Vol. 85, p. 99. Springer, Berlin (1996).

12. H. S. Nalva, H. Kasai, S. Okada, H. Oikawa, H. Matsuda, A. Kakuta, A. Mukoh and H. Nakanishi, Adv. Mater. 5, 758 (1993).

13. I. A. Akimov, I. Denisyuk and A. M. Meshkov, J. Opt. Spectr. 77, 858 (1994).

14. Y. Komai, H. Kasai, H. Hirakoso, Y. Hakuta, H. Katagi, S. Okada, H. Oikawa, T. Adschiri, H. Inomata, K. Arai and H. Nakanishi, Jpn. J. Appl. Phys. 38, L81 (1999).

15. Y. Shen, J. Swiatkiewicz, J. Winiarz and P. Markowicz, P. N. Prasad, Appl. Phys. Lett. 77, 2946 (2000).

16. M. Bienfait and R. Kern, Bull. Soc. Franç. Minér. Crist. 87, 604 (1964).

17. F. Lefaucheux and M. C. Robert, in: D. T. J. Hurle (Ed.), Crystal Growth in Gels, Handbook of Crystal Growth, Vol. 2-b, p.1271. North-Holland, Amsterdam (1994).

18. V. K. La Mer, J. Am. Chem. Soc. 72, 4847 (1950).

19. H. Scholze, J. Non-Cryst. Solids 73, 669 (1985).

20. G. S. He, J. Zieba, J. T. Bradshaw, M. R. Kazmierczak and P. N. Prasad, Opt. Commun. 104, 102 (1993).

21. V. S. Gorelik, A. D. Kudryavtseva, A. I. Sokolovskaya and N. V. Chernega, Opt. Spectrosc. 81, 369 (1996).

22. J. Pécaut, J. P. Lévy and R. Masse, J. Mater. Chem. 3, 999 (1993).

23. S. N. Oliver, P. Pantelis and P. L. Dunn, Appl. Phys. Lett. 56, 307 (1990).

24. P. A. Chollet, V. Dumarcher, J. M. Nunzi, P. Feneyrou and P. L. Baldeck, Nonlin. Opt. 21, 299 (1999).

25. J. Zyss, D. S. Chemla and J. F. Nicoud, J. Chem. Phys. 74, 4800 (1981).

26. S. Bénard and P. Yu, Adv. Mater. 12, 48 (2000).

27. N. Sanz, P. L. Baldeck, J. F. Nicoud, Y. Le Fur and A. Ibanez, J. Solid State Sci. 3, 867 (2001).

28. S. Spagnoli, D. Block, E. Botzung-Appert, I. Colombier, P. L. Baldeck, A Ibanez and A. Corval, J. Phys. Chem. B 109, 8587 (2005).

29. S. Brasselet, V. Le Floc'h, F. Treussart, J. F. Roch, J. Zyss, E. Botzung and A. Ibanez, Phys. Rev. Lett. 92, 207401 (2004).

30. J. Zaccaro, N. Sanz, E. Botzung-Appert, P. L. Baldeck and A. Ibanez, C. R. Phys. 3, 463 (2002).

31. E. Botzung-Appert, J. Zaccaro, C. Gourgon, Y. Usson, P. L. Baldeck and A. Ibanez, J. Cryst. Growth 283, 444 (2005).

32. E. Botzung-Appert, V. Monnier, T. Ha-Duong, R. Pansu and A. Ibanez, Chem. Mater. 16, 1609 (2004). 